\title{
Zinc Chloride Catalyzed Amino Claisen Rearrangement of 1-N-Allylindolines: An Expedient Protocol for the Synthesis of Functionalized 7-Allylindolines
}

https://doi.org/10.1515/hc-2019-0010

Received April 28, 2018; accepted January 16, 2019.

Abstract: 7-Allylindolines are valuable synthons for designing biologically active molecular libraries. Lewis acid catalyzed amino-Claisen rearrangement provides a one pot synthetic entry to these heteroarenes. In this context, Zinc chloride $\left(\mathrm{ZnCl}_{2}\right)$-N,N-dimethylformamide system efficiently catalyzed amino-Claisen rearrangements of 1- $\mathrm{N}$-allylindolines to 7-allylindolines. The rearrangement is influenced by stereoelectronic effects of substituents present in $1-N$-allylindolines. The substrates containing electron donating functionalities underwent rearrangement at lower temperature than substrates with electron withdrawing functional groups. The regioselectivity of the process is governed by the substitution pattern on allyl moiety in $1-N$-allylindoline as well as $\mathrm{ZnCl}_{2}$ catalyst loading in the reaction system.

Keywords: Amino-Claisen rearrangement, $1-\mathrm{N}$-allylindoline, 7-allylindolines, Lewis acid catalysis.

\section{Introduction}

The 7-allylindoline nucleus is prevalent as structural motif for designing indole scaffolds of pharmaceutical importance [1-8]. The Carbon- Carbon bond forming reactions have proven to be the most powerful tool in designing such types of molecular libraries for organic, combinatorial and medicinal chemistry [9-10]. The Claisen rearrangements of vinyl and aryl allyl ether are exploited as an expeditious approach to Carbon-Carbon bond formation in organic syntheses [11-13]. In contrast, the aminoClaisen rearrangement, the nitrogen analogue of Claisen

* Corresponding authors: Seema Jain, Department of Chemistry, GLA University, Mathura, 281406, e-mail: seema.jain.gla@gmail.com rearrangement has attracted little attention due to several limitations including slow rates, the need of higher temperature and low yields due to simultaneous allylic cleavage or cyclization leading to $\mathrm{C}-\mathrm{N}$ bond formation. Therefore, there is the great dearth of examples exists in the literature for amino- Claisen rearrangements in aromatic and heteroaromatic series.

The well documented and classical approach of thermal and uncatalyzed amino Claisen rearrangement of $\mathrm{N}$-allylindolines is suffered from severe drawbacks including thermal decomposition of the substrate and side reactions owing to the requirement of high temperature of $250-280^{\circ} \mathrm{C}$, led to poor yields of 7-allylindolines [14-15]. The limitation associated with the thermal amino-Claisen rearrangement stimulated further interest of synthetic organic chemist for developing milder methods. The strategy of Lewis acid catalysis facilitates the amino Claisen rearrangement of indolines via a charge accelerated process. In this series, the amino- Claisen rearrangement of $\mathrm{N}$-allylindolines has already been reported in different catalytic systems viz in $\mathrm{ZnCl}_{2}$-xylene [16], $\mathrm{BF}_{3} \cdot \mathrm{OEt}_{2}-$ sulfolane [17], $\mathrm{BF}_{3} \cdot \mathrm{OEt}_{2}-$ sulfolane for the synthesis of novel indole analogue of mycophenolic acid as potential antineoplastic agents [18] and in TFA $-\mathrm{CH}_{2} \mathrm{Cl}_{2}$ for the total synthesis of (+)- Okaramine J [19]. Although, these methods are advantageous over uncatalyzed rearrangements for accessing 7-allylindolines, still, some of these methods could not overcome the requirement of higher temperature, longer reaction times, costly catalyst loading in reaction systems and poor yields of targeted molecules. These limitations led us to explore the generality of this important strategic tool for C-C bond formation for targeted molecules from 1-H- $\mathrm{N}$-allylindolines to 7-allylindolines. As a part of our ongoing interest, in Lewis acid catalyzed amino Claisen rearrangement [20-22], we found that $\mathrm{ZnCl}_{2}$ is an efficient catalyst for influencing the reaction. Therefore, $\mathrm{ZnCl}_{2}-\mathrm{N}, \mathrm{N}$-dimethylformamide catalytic system was employed for effecting amino-Claisen rearrangement of

Ә Open Access. @ 2019 Seema Jain, published by De Gruyter. @ - This work is licensed under the Creative Commons Attribution alone 4.0 License. 
$\mathrm{N}$-allylindolines in a view to explore the versatility of the substrates containing electron withdrawing or electron donating functionalities in aryl ring with different patterns of allylic functionality to furnish 7-allylindoline derivatives. The development of reaction presented herein, takes the advantage of an efficient, one pot and regioselective synthetic entry to functionalized 7-allylindolines under very mild reaction conditions. The results emanated from the present study are described herein.

\section{Result and discussion}

A mixture of 1-H- $\mathrm{N}$-allylindoline 1a-g and $\mathrm{ZnCl}_{2}$ (3.0 equiv.) in $\mathrm{N}, \mathrm{N}$-dimethylformamide as a solvent was heated at a temperature range of $120-150^{\circ} \mathrm{C}$ under argon for two hours to furnish $2 \mathbf{~ a - g}$ (Scheme 1) in moderate to good yields. The use of this catalytic system reduced the formation of by products and other tarry products arising due to side reactions as compared to an uncatalyzed thermal process. The result is attributed to the use of polar and aprotic solvent DMF in which $\mathrm{ZnCl}_{2}$ catalyst showed enhanced solubility than any other nonpolar solvent. All reactions produced only a [3,3]-sigmatropic product but under similar conditions the amino Claisen rearrangement of 5-methyl-1- $N$-(3-methyl-2-butenyl)indoline (1h) proceeded slowly to form a mixture of 5-methyl-7-(3-methyl-2-butenyl) indoline (2h) and 1,2,5,6-tetrahydro-4,4,8-trimethyl$4 \mathrm{H}$-pyrrolo[3,2,1-ij] quinoline (3). The formation of $\mathbf{2} \mathbf{h}$ is presumed to be formed from $\mathbf{1 h}$, apparently by the cleavage followed by electrophilic attack of the allylic moiety on the adjacent position of heteroaryl ring without inversion of the allyl group. The failure of $\mathbf{1 h}$ to undergo 3,3-sigmatropic process could be assigned to the steric hindrance on the allyl moiety while passing through the concerted reaction to furnish $\mathbf{2 h}$. The regioisomer $\mathbf{3}$ probably arose due to the acid catalyzed cyclization of $\mathbf{2 h}$ via ionic C-C bond formation (Scheme 1) [16].

However, the formation of $\mathbf{3}$ was effectively inhibited by increasing the amount of $\mathrm{ZnCl}_{2}$ (4.0 equiv.) and shortening the reaction time $(1.0 \mathrm{~h})$ thereby providing the regioselectivity by altering the reaction centre on the substrate to give $\mathbf{2 h}$ in $77 \%$ yield as the sole product. In contrast, 1f, cleanly underwent the rearrangement at lowest temperature to furnish $\mathbf{2 f}$ in good yield ( $86 \%$ ). This result can be attributed to the higher reactivity of the allyl group owing to the steric hindrance in the Lewis acid mediated "charge induced" 3,3-sigmatropic rearrangement process. With ongoing similar problems, 1e reacted faster than $\mathbf{1 g}$ in a 3,3-sigmatropic process to give $\mathbf{2 e}(83 \%)$ and $\mathbf{2 g}$ (77\%) respectively, again giving the evidence for inherited regioselectivity within the reaction process. All products are well characterized by elemental analysis, IR, ${ }^{1} \mathrm{H}$ NMR, ${ }^{13} \mathrm{C}$ NMR and Mass spectral data.

\section{Conclusion}

This rearrangement tolerated electron rich as well as electron deficient substituents on the arene ring of heteroaromatic system without involving the formation of any cyclized product thereby providing excellent regioselectivity to the process. 1-H- $\mathrm{N}$-allylindoline having electron donating groups underwent rearrangement at lower temperature as compared to electron deficient ones in moderate to good yields. The reaction also tolerated acid and base sensitive functionalities on the aryl ring of indoline system under mild reaction conditions. These results are consistent to the proposed mechanism of Lewis acid catalyzed amino Claisen rearrangement via charge accelerated process [20]. Hence, this C-N activation protocol for effecting amino-Claisen rearrangement of 1-H- $\mathrm{N}$-allylindolines provided a one pot, regioselective and an efficient synthetic entry to difficultly accessible 7-allylindolines and should find applicability for creating more sophisticated chemical transformations.

\section{Experimental Details}

1-H- $\mathrm{N}$-allylindolines 1a-h were prepared by using the typical procedure for $\mathrm{N}$-allylation of corresponding 1-H-indolines [23]. 1-H- $\mathrm{N}$-allylindolines $\mathbf{1 a - h}$ furnished corresponding 7-allylindolines $\mathbf{2 a}-\mathbf{h}$ which were characterized on the basis of elemental analysis, IR, ${ }^{1} \mathrm{H},{ }^{13} \mathrm{CNMR}$ and MS data as given below. IR spetra were recorded on Pye Unicam Model SP3-300 infracord in neat. ${ }^{1} \mathrm{H}$ and ${ }^{13} \mathrm{C}$ NMR spectra were recorded on a FT NMR Brucker AM 300L Spectrometer using $\mathrm{CDCl}_{3}$ as solvent and TMS as an internal reference at $300 \mathrm{MHz}$. and $100 \mathrm{MHz}$ respectively. Mass spectra were recorded on a Jeol D-300 (EI) Spectrometer using $\mathrm{CDCl}_{3}$ as solvent.

\section{General procedure for the preparation of 7-allylindolines}

A mixture of 1-H- $N$-allylindolines (1a-h) $(2.57 \mathrm{~g}, 14.9$ $\mathrm{mmol}$ ), $\mathrm{ZnCl}_{2}$ (6.10 g $44.7 \mathrm{mmol}, 3.0$ equiv.) and $\mathrm{DMF}$ $(25 \mathrm{~mL})$ were heated under argon at the temperature 120$150^{\circ} \mathrm{C}$ for $2 \mathrm{hr}$. The mixture was cooled and poured into ice water $(50 \mathrm{~mL})$ and $10 \% \mathrm{HCl}$ solution was added dropwise to make the solution acidic ( $\mathrm{pH} 1)$. The solution was washed with $\mathrm{CHCl}_{3}(2 \times 40 \mathrm{~mL})$, the aqueous layer was 


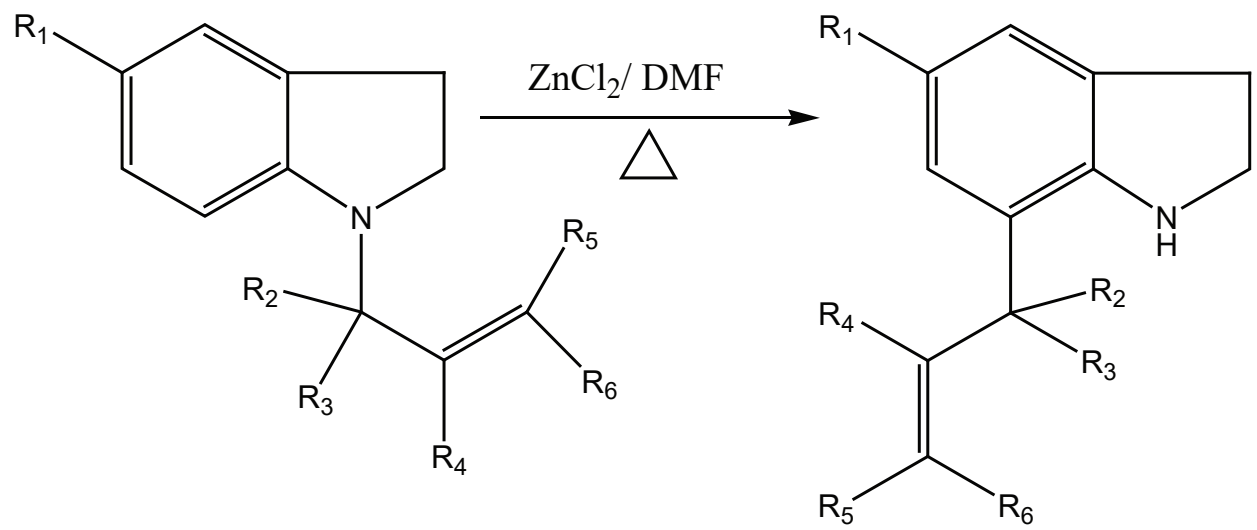

$1 \mathbf{a}-\mathbf{h}$

$2 \mathbf{a}-\mathbf{h}$

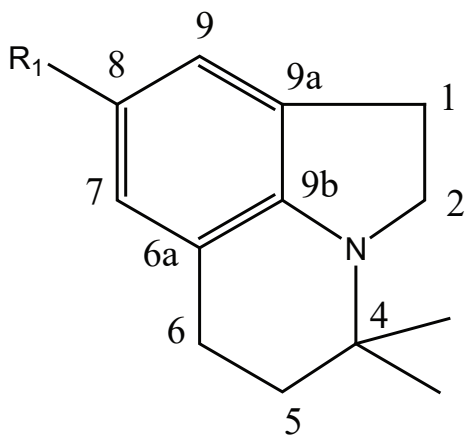

3

Scheme 1 Substrate Scope in Amino Claisen rearrangement.

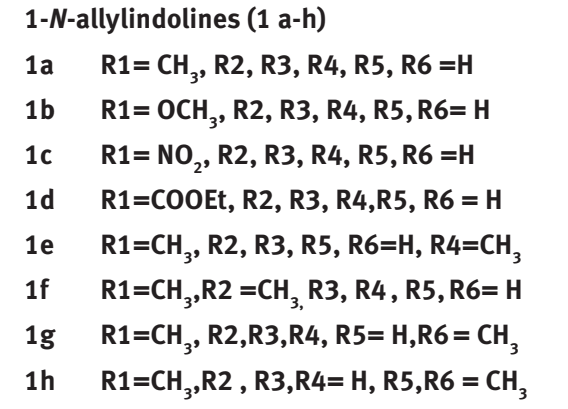

made alkaline with addition of $10 \% \mathrm{NaOH}$ solution and extracted with ether $(3 \times 50 \mathrm{~mL})$. The combined ether extracts were washed with water $(60 \mathrm{~mL})$, dried over $\mathrm{MgSO}_{4}$ and concentrated. The residues were purified by column chromatography on silica gel (hexane : ethyl acetate; 10:1) to afford 7-allylindolines (2a-h).

\section{Analytical data of the compounds:}

7-Allyl-5-methylindoline (2a): The compound was obtained from 1a at the temperature $120-130^{\circ} \mathrm{C}$ in $78 \%$ yield as pale yellow oil; IR (neat) : 3380, 2836, 1615, 1485, 1450,

$$
\begin{aligned}
& \text { 7-Allylindolines (2 a-h) } \\
& \text { 2a } \mathrm{R} 1=\mathrm{CH}_{3}, \mathrm{R} 2, \mathrm{R} 3, \mathrm{R} 4, \mathrm{R} 5, \mathrm{R} 6=\mathrm{H} \\
& \text { 2b R1 }=\mathrm{OCH}_{3}, \mathrm{R} 2, \mathrm{R} 3, \mathrm{R} 4, \mathrm{R} 5, \mathrm{R} 6=\mathrm{H} \\
& \text { 2c } R 1=\mathrm{NO}_{2}, \mathrm{R2}, \mathrm{R3}, \mathrm{R} 4, \mathrm{R} 5, \mathrm{R} 6=\mathrm{H} \\
& \text { 2d R1=COOEt, R2, R3, R4, R5, R6 =H } \\
& \text { 2e } \mathrm{R} 1=\mathrm{CH}_{3}, \mathrm{R} 2, \mathrm{R} 3, \mathrm{R} 5, \mathrm{R} 6=\mathrm{H}, \mathrm{R} 4=\mathrm{CH}_{3} \\
& \text { 2f } \quad \mathrm{R}_{1}=\mathrm{CH}_{3}, \mathrm{R} 2, \mathrm{R} 3, \mathrm{R} 4, \mathrm{R} 6=\mathrm{H}, \mathrm{R} 5=\mathrm{CH}_{3} \\
& \mathrm{R} 1=\mathrm{CH}_{3}, \mathrm{R} 2=\mathrm{CH}_{3}, \mathrm{R} 3, \mathrm{R} 4, \mathrm{R} 5, \mathrm{R} 6=\mathrm{H} \\
& \mathrm{R} 1=\mathrm{CH}_{3}, \mathrm{R} 2, \mathrm{R} 3, \mathrm{R} 4=\mathrm{H}, \mathrm{R} 5, \mathrm{R} 6=\mathrm{CH}_{3}
\end{aligned}
$$

1250, 995, $910 \mathrm{~cm}^{-1}$; ${ }^{1} \mathrm{H}$ NMR: $\delta 3.15\left(\mathrm{t}, J=8.2 \mathrm{~Hz}, 2 \mathrm{H}, \mathrm{C}_{3}-\mathrm{H}\right)$, $3.41\left(\mathrm{dd}, J=6.1 \mathrm{~Hz}, J=1.2 \mathrm{~Hz}, 2 \mathrm{H},-\mathrm{CH}_{2}-\mathrm{C}=\mathrm{C}\right), 3.66-3.91(\mathrm{~m}$ $3 \mathrm{H}, \mathrm{NH}$ and $\left.\mathrm{C}_{2} \mathrm{H}\right), 5.26-5.30\left(\mathrm{~m}, 2 \mathrm{H},-\mathrm{C}=\mathrm{CH}_{2}\right)$, 6.04- 6.17 $(\mathrm{m}, 1 \mathrm{H},-\mathrm{C}-\mathrm{CH}=\mathrm{C}), 6.60-7.20(\mathrm{~m}, 2 \mathrm{H}, \mathrm{Ar}-\mathrm{H}), 2.60(\mathrm{~s}, 3 \mathrm{H}$, Ar- $\left.\mathrm{CH}_{3}\right)$; ${ }^{13} \mathrm{C}$ NMR: $\delta 146.6(\mathrm{C} 8), 135.6\left(-\mathrm{CH}=\mathrm{CH}_{2}\right), 127.3(\mathrm{C} 6)$, 126.3 (C9), 126.5 (C4), 125.6 (C5), 121.9 (C7), 116.1 (-C= $\left.\mathrm{CH}_{2}\right)$, 46.5 (C2), $38.3\left(\mathrm{Ar}^{-} \mathrm{CH}_{2}\right), 30.4(\mathrm{C} 3), 23.6\left(\mathrm{Ar}^{-} \mathrm{CH}_{3}\right)$; MS: $\mathrm{m} / \mathrm{z}$ $173\left(\mathrm{M}^{+}\right)$; Anal. Calcd for $\mathrm{C}_{12} \mathrm{H}_{15} \mathrm{~N}$ : C, 83.23; $\mathrm{H}, 8.67 ; \mathrm{N}, 8.09$. Found: C, 83.26; H, 8.98; N, 8.06.

7-Allyl-5-methoxyindoline (2b): The compound was obtained from $\mathbf{1 b}$ at $130-135^{\circ} \mathrm{C}$ in $62 \%$ yield as brown oil; IR (neat) : 3382, 2936, 2832, 1615, 1485, 1460, 1246, 990, 
$900 \mathrm{~cm}^{-1} ;{ }^{1} \mathrm{H}$ NMR : $\delta 2.99\left(\mathrm{t}, J=8.4 \mathrm{~Hz}, 2 \mathrm{H}, \mathrm{C}_{3}-\mathrm{H}\right), 3.33$ $\left(\mathrm{dd}, J=6.3 \mathrm{~Hz}, J=1.2 \mathrm{~Hz}, 2 \mathrm{H},-\mathrm{CH}_{2}-\mathrm{C}=\mathrm{C}\right), 3.57-3.78(\mathrm{~m} 3 \mathrm{H}$, $\mathrm{NH}$ and $\left.\mathrm{C}_{2} \mathrm{H}\right), 5.01-5.09\left(\mathrm{~m}, 2 \mathrm{H},-\mathrm{C}=\mathrm{CH}_{2}\right)$, 5.97- $6.27(\mathrm{~m}, 1 \mathrm{H}$, $-\mathrm{C}-\mathrm{CH}=\mathrm{C}), 6.70-7.19(\mathrm{~m}, 2 \mathrm{H}, \mathrm{Ar}-\mathrm{H}), 3.78\left(\mathrm{~s}, 3 \mathrm{H}, \mathrm{Ar}-\mathrm{OCH}_{3}\right) ;{ }^{13} \mathrm{C}$ NMR : $\delta 147.8$ (C5), $141.6(\mathrm{C} 8), 135.6\left(\mathrm{CH}=\mathrm{CH}_{2}\right), 127.7$ (C9), 122.1 (C7), $116.3\left(\mathrm{C}=\mathrm{CH}_{2}\right), 115.4$ (C6), 108.9 (C4), 47.2 (C2), $38.8\left(\mathrm{Ar}^{-} \mathrm{CH}_{2}\right), 31.2(\mathrm{C} 3), 29.6\left(\mathrm{Ar}^{-} \mathrm{CH}_{3}\right)$; $\mathrm{MS}: \mathrm{m} / \mathrm{z} 189\left(\mathrm{M}^{+}\right)$; Anal. Calcd for $\mathrm{C}_{12} \mathrm{H}_{15} \mathrm{NO}$ : C, 76.19; H, 7.94; N, 7.40. Found: C, 76.08; H, 7.98; N, 7.36.

7-Allyl-5-nitroindoline (2c): The compound was obtained from 1c at $140-150^{\circ} \mathrm{C}$ in $80 \%$ yield as yellow oil; IR (neat) : 3432, 3386, 2960, 2860, 1632, 1594, 1520, 1260, 1456, 1441, 1350, 1309, 1275, $995910 \mathrm{~cm}^{-1}$; ${ }^{1} \mathrm{H}$ NMR : $\delta 3.11(\mathrm{t}, J=$ $\left.8.2-8.4 \mathrm{~Hz}, 2 \mathrm{H}, \mathrm{C}_{3}-\mathrm{H}\right), 3.42(\mathrm{dd}, J=6.0 \mathrm{~Hz}, J=1.2 \mathrm{~Hz}, 2 \mathrm{H}$, $\left.-\mathrm{CH}_{2}-\mathrm{C}=\mathrm{C}\right)$, 3.66- $4.01\left(\mathrm{~m} \mathrm{3H}, \mathrm{NH}\right.$ and $\left.\mathrm{C}_{2} \mathrm{H}\right), 5.07-5.15(\mathrm{~m}$, $\left.2 \mathrm{H},-\mathrm{C}=\mathrm{CH}_{2}\right)$, 5.91- $6.02(\mathrm{~m}, 1 \mathrm{H},-\mathrm{C}-\mathrm{CH}=\mathrm{C}), 7.05-7.28(\mathrm{~m}, 2 \mathrm{H}$, Ar-H); ${ }^{13} \mathrm{C}$ NMR : $\delta 155.3$ (C8), $135.6(\mathrm{C} 5), 135.4\left(-\mathrm{CH}=\mathrm{CH}_{2}\right)$, 128.6 (C9), 122.6 (C7), 121.2 (C6), 119.6 (C4), $116.9\left(-\mathrm{C}=\mathrm{CH}_{2}\right)$, 46.1 (C2), $37.8\left(\mathrm{Ar}^{\left.-\mathrm{CH}_{2}\right)}\right.$, 29.3 (C3); MS : m/z $204\left(\mathrm{M}^{+}\right)$; Anal. Calcd for $\mathrm{C}_{11} \mathrm{H}_{12} \mathrm{~N}_{2} \mathrm{O}_{2}:$ C, 64.71; H, 5.88; N, 13.73. Found: C, 64.74; H, 5.80; N, 13.76 .

7-Allyl-5-carbethoxyindoline (2d): The compound was obtained from $1 d$ at $135-145^{\circ} \mathrm{C}$ in $79 \%$ yield as pale yellow oil; IR (neat) : 3486, 3372, 2958, 1690, 1620, 1586, 1570, 1456, 1432, 1310, 1280, 1248, 1200, 1085, 1070, 985, 900 $\mathrm{cm}^{-1} ;{ }^{1} \mathrm{H}$ NMR : $\delta 3.18\left(\mathrm{t}, J=8.1 \mathrm{~Hz}, 2 \mathrm{H}, \mathrm{C}_{3}-\mathrm{H}\right), 3.42(\mathrm{~d}, J=1.0$ $\left.\mathrm{Hz}, 2 \mathrm{H},-\mathrm{CH}_{2}-\mathrm{C}=\mathrm{C}\right)$, 3.65- $3.86\left(\mathrm{~m} \mathrm{3H}, \mathrm{NH}\right.$ and $\left.\mathrm{C}_{2} \mathrm{H}\right)$, 5.08$5.10\left(\mathrm{~m}, 2 \mathrm{H},-\mathrm{C}=\mathrm{CH}_{2}\right), 6.05-6.75(\mathrm{~m}, 1 \mathrm{H},-\mathrm{C}-\mathrm{CH}=\mathrm{C}), 6.20-6.89$ (m, 2H, Ar-H), 2.56 (q, $\left.J=8.1 \mathrm{~Hz}, 2 \mathrm{H}, \mathrm{COOCH}_{2}-\mathrm{C}\right), 1.14$ (t, $J$

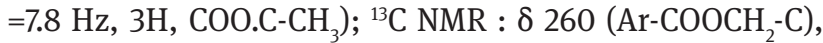
166 (Ar-COOC-C), 154.3(C8), 135.8 (- $\left.\mathrm{CH}=\mathrm{CH}_{2}\right), 127.3$ (C6), 126.6 (C9), 125.7 (C4), 121.4 (C7), 117.5 (C5), $116.3\left(-\mathrm{C}=\mathrm{CH}_{2}\right)$, 46.8 (C2), $38.9\left(\mathrm{Ar}^{-} \mathrm{CH}_{2}\right)$, 29.9 (C3); MS : m/z $204\left(\mathrm{M}^{+}\right)$; Anal. Calcd for $\mathrm{C}_{11} \mathrm{H}_{12} \mathrm{~N}_{2} \mathrm{O}_{2}: \mathrm{C}, 64.71 ; \mathrm{H}, 5.88 ; \mathrm{N}, 13.73$. Found: C, 64.74; H, 5.80; N, 13.76.

5-Methyl-7-(2-methylallyl)indoline (2e): The compound was obtained from 1 e at $130-140^{\circ} \mathrm{C}$ in $83 \%$ yield as pale yellow oil; IR (neat) : 3375, 2840, 1640, 1485, 1450, 1250, $910 \mathrm{~cm}^{-1}$; ${ }^{1} \mathrm{H}$ NMR : $\delta 3.17$ (t, $J=8.1 \mathrm{~Hz}, 2 \mathrm{H}$, $\left.\mathrm{C}_{3}-\mathrm{H}\right), 3.46\left(\mathrm{dd}, J=6.8 \mathrm{~Hz}, J=1.4 \mathrm{~Hz}, 2 \mathrm{H},-\mathrm{CH}_{2}-\mathrm{C}=\mathrm{C}\right), 3.60-$ $3.80\left(\mathrm{~m}, 3 \mathrm{H}, \mathrm{NH}\right.$ and $\left.\mathrm{C}_{2} \mathrm{H}\right), 5.26-5.30(\mathrm{dd}, J=18 \mathrm{~Hz}, J=$ $\left.2 \mathrm{~Hz}, 2 \mathrm{H},-\mathrm{C}=\mathrm{CH}_{2}\right), 1.86$ (s, 3H, $\left.-\mathrm{C}-\mathrm{C}\left(\mathrm{CH}_{3}\right)=\mathrm{C}\right), 6.60-7.20$ (m, 2H, Ar-H), 2.58 (s, 3H, Ar- $\left.\mathrm{CH}_{3}\right) ;{ }^{13} \mathrm{C} \mathrm{NMR} \mathrm{:} 146.8$ (C8), $142.8\left(\mathrm{CH}=\mathrm{CH}_{2}\right), 127.7$ (C6), 126.6 (C9), 126.4 (C4), 125.3 (C5), 121.9 (C7), $111.3\left(-\mathrm{C}=\mathrm{CH}_{2}\right), 46.8$ (C2), $38.9\left(\mathrm{Ar}^{-} \mathrm{CH}_{2}\right)$, 30.8 (C3), $24.9\left(\mathrm{Ar}^{-} \mathrm{CH}_{3}\right), 22.1\left(\mathrm{CH}_{3}-\mathrm{CH}=\mathrm{C}\right)$; $\mathrm{MS}: \mathrm{m} / \mathrm{z} 187$ $\left(\mathrm{M}^{+}\right)$; Anal. Calcd for $\mathrm{C}_{11} \mathrm{H}_{12} \mathrm{~N}_{2} \mathrm{O}_{2}$ : C, 83.32; H, 90.91; N, 7.49. Found: C, 83.39; H, 90.87; N, 7.51.

5-Methyl-7-(trans-2-butenyl)indoline (2f): The compound was obtained from 1f at $120-130^{\circ} \mathrm{C}$ in $86 \%$ yield as pale yellow oil; IR (neat) : 3386, 3050, 2945, 2847, 1600, 1460, 1255, $970 \mathrm{~cm}^{-1} ;{ }^{1} \mathrm{H}$ NMR : $\delta: 3.18(\mathrm{t}, J=8.2-8.4 \mathrm{~Hz}, 2 \mathrm{H}$, $\left.\mathrm{C}_{3}-\mathrm{H}\right), 3.48\left(\mathrm{dd}, J=6.6 \mathrm{~Hz}, J=1.2 \mathrm{~Hz}, 2 \mathrm{H},-\mathrm{CH}_{2}-\mathrm{C}=\mathrm{C}\right), 3.66-$ $3.80\left(\mathrm{~m}, 3 \mathrm{H}, \mathrm{NH}\right.$ and $\left.\mathrm{C}_{2} \mathrm{H}\right), 6.02-6.19(\mathrm{~m}, 1 \mathrm{H},-\mathrm{C}-\mathrm{CH}=\mathrm{C})$, 5.10-5.30 (m, $1 \mathrm{H},-\mathrm{C}=\mathrm{CH}-\mathrm{C}), 1.64(\mathrm{~d}, J=18 \mathrm{~Hz}, 3 \mathrm{H},-\mathrm{C}=\mathrm{C}-$ $\left.\mathrm{CH}_{3}\right), 6.80-7.19(\mathrm{~m}, 2 \mathrm{H}, \mathrm{Ar}-\mathrm{H}), 2.50\left(\mathrm{~s}, 3 \mathrm{H}, \mathrm{Ar}-\mathrm{CH}_{3}\right) ;{ }^{13} \mathrm{C} \mathrm{NMR}$ : $\delta 146.6$ (C8), 127.6 (C6), 126.5 ( $\left.\mathrm{CH}=\mathrm{CH}_{2}\right), 126.4$ (C9), 126.1 (C4), $125.3\left(-\mathrm{C}=\mathrm{CH}_{2}\right), 125.2$ (C5), 121.9 (C7), 46.9(C2), 38.8 $\left(\mathrm{Ar}-\mathrm{CH}_{2}\right), 30.9$ (C3), 24.2( $\left.\mathrm{Ar}-\mathrm{CH}_{3}\right), 18.3\left(=\mathrm{CH} \mathrm{CH}_{3}\right) ; \mathrm{MS}: \mathrm{m} / \mathrm{z}$ $187\left(\mathrm{M}^{+}\right)$; Anal. Calcd for $\mathrm{C}_{11} \mathrm{H}_{12} \mathrm{~N}_{2} \mathrm{O}_{2}$ : C, 83.42; $\mathrm{H}, 9.09$; N, 7.49. Found: C, 83.40; H, 9.12; N, 7.51.

5-Methyl-7-(1-methylallyl)indoline (2g): The compound was obtained from $1 \mathrm{~g}$ at $130-140^{\circ} \mathrm{C}$ in $77 \%$ yield as pale yellow oil; IR (neat) : 3380, 2840, 1640, 1480, 1440, 1250, 990, $910 \mathrm{~cm}^{-1}$; ${ }^{1} \mathrm{H}$ NMR : $\delta 3.16\left(\mathrm{t}, J=8.2 \mathrm{~Hz}, \mathrm{C}_{3}-\mathrm{H}\right), 2.98(\mathrm{~m}$, $1 \mathrm{H},-\mathrm{CH}-\mathrm{C}=\mathrm{C}), 1.67$ (dd, $J=6.5 \mathrm{~Hz}, J=1.4 \mathrm{~Hz} 3 \mathrm{H},-\mathrm{C}\left(\mathrm{CH}_{3}\right)$ $\mathrm{C}=\mathrm{C})$, 3.65- $3.86\left(\mathrm{~m} \mathrm{3H}, \mathrm{NH}\right.$ and $\left.\mathrm{C}_{2} \mathrm{H}\right), 5.12-5.30(\mathrm{~m}, 2 \mathrm{H}$, $\left.-\mathrm{C}=\mathrm{CH}_{2}\right), 6.05-6.15(\mathrm{~m}, 1 \mathrm{H},-\mathrm{C}-\mathrm{CH}=\mathrm{C}), 6.60-7.00(\mathrm{~m}, 2 \mathrm{H}$, Ar-H), 2.60 (s, 3H, Ar- $\left.\mathrm{CH}_{3}\right) ;{ }^{13} \mathrm{C}$ NMR : $\delta 144.6$ (C8), 138.2 $\left(\mathrm{CH}=\mathrm{CH}_{2}\right), 127.9$ (C7), 127.3 (C6), 126.8 (C4), 126.3 (C9), 125.4 (C5), $115.3\left(-\mathrm{C}=\mathrm{CH}_{2}\right), 46.8(\mathrm{C} 2), 38.4\left(\mathrm{Ar}^{-} \mathrm{CH}_{2}\right), 31.2(\mathrm{C} 3), 24.4$ $\left(\mathrm{Ar}-\mathrm{CH}_{3}\right), 20.9\left(\mathrm{Ar}-\mathrm{CH} . \mathrm{CH}_{3}\right)$; MS : m/z $186\left(\mathrm{M}^{+}\right)$; Anal. Calcd. for $\mathrm{C}_{11} \mathrm{H}_{12} \mathrm{~N}_{2} \mathrm{O}_{2}: \mathrm{C}, 83.87 ; \mathrm{H}, 8.60 ; \mathrm{N}$, 7.53. Found: C, 83.91; $\mathrm{H}$, $8.58 ; \mathrm{N}, 7.50$.

5-Methyl-7-(3-methyl-2-butenyl)indoline (2h): The compound was obtained from $1 \mathrm{~h}$ at $140-150^{\circ} \mathrm{C}$ in $78 \%$ yield as yellow oil; IR (neat) : 3380, 3045, 2970, 2850, 1670, 1625, 1455, 1260, $835 \mathrm{~cm}^{-1}$; ${ }^{1} \mathrm{H}$ NMR : $\delta: 3.10(\mathrm{t}, \mathrm{J}=8.2-8.4 \mathrm{~Hz} 2 \mathrm{H}$, $\left.\mathrm{C}_{3}-\mathrm{H}\right), 3.43\left(\mathrm{dd}, J=6.1 \mathrm{~Hz}, J=1.2 \mathrm{~Hz} 2 \mathrm{H},-\mathrm{CH}_{2}-\mathrm{C}=\mathrm{C}\right), 3.50-3.60$ ( $\mathrm{m}, 3 \mathrm{H}, \mathrm{NH}$ and $\left.\mathrm{C}_{2} \mathrm{H}\right), 5.24(\mathrm{t}, J=2.1 \mathrm{~Hz} 1 \mathrm{H},-\mathrm{C}-\mathrm{CH}=\mathrm{C}), 1.91$ [s, 6H, $\left.-\mathrm{C}-\mathrm{C}=\mathrm{C}\left(\mathrm{CH}_{3}\right)_{2}\right], 6.61-7.16(\mathrm{~m}, 2 \mathrm{H}, \mathrm{Ar}-\mathrm{H}), 2.50(\mathrm{~s}, 3 \mathrm{H}$, $\left.\mathrm{Ar}-\mathrm{CH}_{3}\right) ;{ }^{13} \mathrm{C} \mathrm{NMR}: \delta 146.6(\mathrm{C} 8), 131.8\left[=\mathbf{C}\left(\mathrm{CH}_{3}\right)_{2}\right], 127.6(\mathrm{C} 6)$, 127.4 (C9), 126.8 (C4), 125.4 (C5), $122.6\left[-\mathrm{CH}=\mathrm{C} .\left(\mathrm{CH}_{3}\right)_{2}\right], 121.8$ (C7), $47.1(\mathrm{C} 2), 32.6\left(\mathrm{Ar}^{-} \mathrm{CH}_{2}\right), 30.8(\mathrm{C} 3), 24.6\left[-\mathrm{C}-\mathrm{C}=\mathrm{C}\left(\mathrm{CH}_{3}\right)_{2}\right]$, $23.8\left(\mathrm{Ar}^{-\mathrm{CH}_{3}}\right), 18.8\left[-\mathrm{C}-\mathrm{C}=\mathrm{C}\left(\mathrm{CH}_{3}\right)_{2}\right]$; $\mathrm{MS}: \mathrm{m} / \mathrm{z} 201\left(\mathrm{M}^{+}\right)$; Anal. Calcd for $\mathrm{C}_{11} \mathrm{H}_{12} \mathrm{~N}_{2} \mathrm{O}_{2}$ : C, 83.58; H, 9.45; N, 6.97. Found: C, 83.60; H, 9.44; N, 6.99 .

1,2,5,6-Tetrahydro-4,4,8-trimethyl-4H-pyrrolo[3,2,1ij]quinoline (3): The compound was obtained from $\mathbf{~ h h}$ at $140-150^{\circ} \mathrm{C}$ in $15 \%$ yield as pale yellow oil; IR (neat) : 3380 , 3050, 2875, 2860, 1670, 1630, 1460, $1250 \mathrm{~cm}^{-1} ;{ }^{1} \mathrm{H}$ NMR : $\delta$ $2.93\left(\mathrm{t}, J=8.3 \mathrm{~Hz}, 2 \mathrm{H}, \mathrm{C}_{3}-\mathrm{H}\right), 2.82-2.89\left(\mathrm{~m} 2 \mathrm{H},-\mathrm{CH}_{2}-\mathrm{C}-\mathrm{C}\right)$, $3.58\left(\mathrm{t}, J=8.1 \mathrm{~Hz} 2 \mathrm{H}, \mathrm{C}_{2}-\mathrm{H}\right), 2.62\left(\mathrm{t}, J=8 \mathrm{~Hz} 2 \mathrm{H},-\mathrm{C}^{-\mathrm{CH}_{2}-}\right.$ C), 1.07 [s, 6H, -C-C-C( $\left.\left(\mathrm{CH}_{3}\right)_{2}\right], 6.49-6.86(\mathrm{~m}, 2 \mathrm{H}, \mathrm{Ar}-\mathrm{H}), 2.58$ (s, 3H, Ar- $-\mathrm{CH}_{3}$ ); ${ }^{13} \mathrm{C}$ NMR : $\delta 145.7$ (9b), 127.6 (9a), 127.7 (C7), 126.3 (C9), 126.8 (C5), 121.9 (6a), 47.9 (C2), 29.3 (C3), 28.9 (C6), $24.9\left[-\mathrm{C}-\mathrm{C}=\mathrm{C}\left(\mathrm{CH}_{3}\right)_{2}\right], 24.9\left[-\mathrm{C}-\mathrm{C}=\mathrm{C}\left(\mathbf{C H}_{3}\right)_{2}\right], 24.1$ (Ar- $\mathrm{CH}_{3}$ ), 22.0 (C5); MS : m/z $201\left(\mathrm{M}^{+}\right)$; Anal. Calcd for $\mathrm{C}_{11} \mathrm{H}_{12} \mathrm{~N}_{2} \mathrm{O}_{2}: \mathrm{C}, 83.58 ; \mathrm{H}, 9.45 ; \mathrm{N}, 6.97$. Found: C, 83.56; H, 9.41; N, 6.96. 


\section{References}

[1] Sharma, V.; Kumar, P.; Pathak, D. Biological Importance of the Indole Nucleus in Recent Years: A Comprehensive Review. J. Heterocycl. Chem. 2010, 47, 491-502.

[2] Kochanowska-Karamyan, A.T.; Hamann, M.T. Marine Indole Alkaloids: Potential in New Drug Leads for the Control of Depression and Anxiety. Chem. Rev. 2010, 110, 4489-4497.

[3] Ruiz- Sachis, P.; Savina, S.A.; Albericio, F.; Alvarez, M. Structure, Bioactivity and Synthesis of Natural Products with Hexahydropyrrolo[2,3-b]indole. Chem-Eur.J. 2011, 17, 1388-1408.

[4] Zhang, Y.; Hubbard, J.W.; Akhmedov, N.G.; Petersen, J.L.; Söderberg, B.C.G. Total Synthesis of the Tetracyclic Indole Alkaloid Ht-13-B. J. Org. Chem. 2015, 80, 4783-4790.

[5] Chaudhuri, S.; Ghosh, S.; Bhunia, S.; Bisai, A. Catalytic Asymmetric formal Total syntheses of (+)- and (-)-Cycloclavine. Chem. Commun. 2018, 54, 940-943.

[6] Lv, J.; Wang, B.; Yuan, K.; Wang, Y.; Jia, Y. Regioselective Direct C4 Functionalization of Indole: Total Syntheses of (-)-Agroclavine and (-)-Elymoclavine. Org. Lett. 2017, 19, 3664-3667.

[7] Tong, S.; Xu, Z.; Mamboury, M.; Wang, Q.; Zhu, J. Aqueous Titanium Trichloride Promoted Reductive Cyclization of o-Nitrostyrenes to Indoles: Development and Application to the Synthesis of Rizatriptan and Aspidospermidine. Angew. Chem. Int. Ed. 2015, 54, 11809-11812.

[8] Zhang, Y.; McArdle, I. W.; Hubbard, J.W.; Akhmedov, N.G.; Söderberg, B.C.G. Total synthesis of the Tetracyclic Indole Alkaloid ht-13-A. Tetrahedron Lett. 2016, 57, 2865-2867.

[9] Horike, S.; Dinca, M.; Tamaki, K.; Long, J.R. Size-Selective Lewis Acid Catalysis in A Microporous Metal-Organic Framework with Exposed $\mathrm{Mn}^{2+}$ Coordination Sites. J. Am. Chem. Soc. 2008, 130, 5854-5855.

[10] Nair, V.; Vellalath, S.; Babu, B.P.; Recent advances in carboncarbon bond-forming reactions involving homoenolates generated by NHC catalysis. Chem.Soc. Rev. 2008, 37, 2691-2698.

[11] Castro Ana, M. Claisen Rearrangement over the Past Nine Decades. Chem. Rev. 2004, 104, 2939-3002.
[12] Nubbemeyer, U. Recent Advances in Charge-Accelerated Aza-Claisen Rearrangements. Top Curr. Chem. 2005, 244, 149 - 213.

[13] Wang, H.Y.; Mueller, D.S.; Sachwani, R.M.; Londino, H.N.; Anderson, L.L. Carbon-Carbon Bond Formation and Pyrrole Synthesis via the $[3,3]$ Sigmatropic Rearrangement of $O$-Vinyl Oxime Ethers. Org. Lett. 2010, 12, 2290-2293.

[14] Cope, A.C.; Hardy, E.M. The Introduction of Substituted Vinyl Groups. V. A Rearrangement Involving the Migration of an Allyl Group in a Three-Carbon System. J. Am. Chem. Soc. 1940, 62, 441-444.

[15] Claisen, L. Ber. Dtsch. Chem. Ges. Claisen Rearrangement. 1912, 45, 3157-3166.

[16] Izumi, T.; Kasahara, A. The acid-catalyzed rearrangement of 1-N-allylindolines. J. Heterocycl. Chem. 1990, 27, 1173-1175.

[17] Anderson W.K.; Lai, G. Boron Trifluoride-Diethyl Ether Complex Catalyzed Aromatic Amino- Claisen Rearrangements. Synthesis, 1995, 1287-1290.

[18] Lai, G.; Anderson, W.K.; Synthesis of Novel Indole Analogues of Mycophenolic Acid as Potential Antineoplastic Agents. Tetrahedron 2000, 56, 2583-2590.

[19] Roe, J.M.; Webster, R.A.B.; Ganesan, A. Total Synthesis of (+)-Okaramine J Featuring an Exceptionally Facile N-Reverseprenyl to C-Prenyl Aza-Claisen Rearrangement. Org. Lett. 2003, 5, 2825-2827.

[20] Jain, S.; Pandey, N.; Kishore, D. Lewis acid catalyzed amino-Claisen rearrangement: A facile one pot synthesis of 2-allylarylamines from $\mathrm{N}$ - allylarylamines. Indian J. Chem. 2007, 46 B, 529-31.

[21] Jain, S.; Pandey, N.; Kishore, D., Proton Catalyzed AminoClaisen Rearrangement: A Practical One Step Approach to the Synthesis of 2-Allylarylamines from N-Allylarylamines. J. Indian Chem. Soc. 2006, 83, 938-940.

[22] Sharma, P.; Kaur, N.; Jain, S.; Kishore, D. Amino-Claisen rearrangment of $\mathrm{N}$-allyl aryl amines: a versatile precursor in the palladium catalyzed hetroannultation to indoles. J. Curr.Chem. Pharm.Sc. 2013, 3, 80-89.

[23] Jain, S. ; Pandey, N.; Kishore, D. An Improved Method of Synthesis of precursors for Amino-Claisen Rearrangement. J. Indian Chem. Soc. 2006, 83, 1052-1056. 